\title{
The identification and development of Canadian coronary artery bypass graft surgery quality indicators
}

\author{
Veena Guru, MD, ${ }^{a}$ Geoffrey M. Anderson, ${ }^{\text {a }}$ Stephen E. Fremes, MD, ${ }^{a}$ Gerald T. O'Connor, PhD, ${ }^{\text {b }}$ Frederick L. Grover, MD, ${ }^{c}$
} Jack V. Tu, MD, PhD, and the Canadian CABG Surgery Quality Indicator Consensus Panel

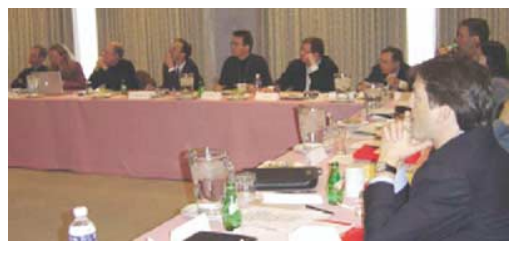

Canadian CABG Surgery Quality Indicator Consensus Panel

\section{eonly Additional material is available online.}

From the Institute For Clinical Evaluative Sciences and Sunnybrook and Women's College Health Sciences Centre, University of Toronto, a Toronto, Canada; Professor, Medicine, Community and Family Medicine, Center for the Evaluative Clinical Sciences, $^{\text {b }}$ Dartmouth Medical School, Hanover, NH; and Division of Cardiovascular Surgery, University of Colorado, ${ }^{\mathrm{c}}$ Denver, Colo.

Funded by the Canadian Institutes of Health Research (CIHR) (fellowship salary support), TACTICS training grant, and the Sunnybrook and Women's College Health Sciences Centre (Tanna Schulich scholarship). This project was supported by the Heart and Stroke Foundation of Ontario HSF 5484 (www.qualitycabg.org).

Received for publication March 25, 2005; revisions received May 2, 2005; accepted for publication July 22, 2005.

Address for reprints: Veena Guru, MD, Institute for Clinical Evaluative Sciences, 2075 Bayview Avenue, G106, Toronto, Ontario M4N 3M5, Canada (E-mail: veena.guru@utoronto.ca).

J Thorac Cardiovasc Surg 2005;130:1257-64 $0022-5223 / \$ 30.00$

Copyright (C) 2005 by The American Association for Thoracic Surgery

doi:10.1016/j.jtcvs.2005.07.041
Objective: The study objective was to develop quality indicators for coronary artery bypass graft surgery that relate to quality of care, associate with preventable death, and could be reported on performance reports.

Methods: A comprehensive list of quality indicators was collected from quality improvement organizations including the Society For Thoracic Surgery, Northern New England Cardiovascular Disease Study Group, and Veteran's Affairs System. Indicators were collated from practice guidelines from the American College of Cardiology and the American Heart Association. A MEDLINE search using the keywords "quality indicators" and "coronary bypass" was completed. A 17-member multidisciplinary international expert panel was assembled, who voted using a 2-step Delphi process regarding association with quality of care, risk adjustment, association with preventable death, and inclusion on performance reports.

Results: A total of 149 quality indicators were examined. This list was distilled to 33 indicators related to quality of care, 10 indicators that could be adequately risk adjusted, 34 indicators related to preventable death, and 18 indicators to be included on performance reports. These selected indicators consisted of 19 outcome variables, 23 process of care variables, and 4 structure variables. The quality indicators believed to be useful on a Canadian institutional coronary artery bypass graft surgery report card included the following: 30-day mortality, in-hospital mortality, electrocardiographic myocardial infarction, red cell transfusion, allogeneic blood product transfusion, deep sternal wound infection, postoperative stroke, postoperative dialysis, intensive care unit readmission, intensive care unit length of stay, ventilation time, repeat cardiac operation, repeat surgery with cardiopulmonary bypass, repeat revascularization, waiting time to surgery, completion of surgery within a recommended waiting time, use of left internal thoracic artery graft, and institutional volume.

Conclusions: This set of consensus quality indicators can be used as a standard list to be monitored by providers of coronary artery bypass graft surgery in an effort to continuously evaluate and improve their performance.

$\mathrm{T}$ The monitoring of quality of care has been an essential part of ongoing quality of care efforts in cardiac surgery. Risk-adjusted mortality is used as the standard measure of quality of care to evaluate the institutional performance for coronary artery bypass graft (CABG) surgery, despite the fact it has an uncertain link to quality of care. ${ }^{1}$ It has been suggested that the publication of hospital mortality may deliver the wrong message to the public about the relative performance of a hospital, because the public is not aware of the weak evidence to date that links mortality to quality of care. ${ }^{2}$

Quality of care as defined by the Institute of Medicine is the "degree to which health services for individuals increases the likelihood of desired health outcomes 


\author{
Abbreviations and Acronyms \\ $\mathrm{CABG}=$ coronary artery bypass graft \\ $\mathrm{ECG}=$ electrocardiogram (electrocardiographic) \\ ICU = intensive care unit \\ $\mathrm{NQF}=$ National Quality Forum
}

and are consistent with current professional knowledge."3 For many aspects of cardiac surgical practice the health services that increase the likelihood of desired health outcomes are not clear. It is also apparent that health outcomes themselves are not adequate to measure quality of care, because they can vary with patient factors and random chance, which cannot always be accounted in statistical modeling.

Report cards have focused on the outcomes portion of the Donabedian model of quality of care consisting of the dimensions of structure, process, and outcome. Structure includes variables related to the resources and systems within a hospital, such as staffing ratios. Process relates to recognized therapeutic management of a patient, for example, the use of internal thoracic artery grafts to bypass the left anterior descending artery. Outcomes represent adverse clinical events that occur after the completion of $\mathrm{CABG}$ surgery.

The argument for using outcomes despite scarce evidence of the link to quality performance is that the goal of clinical care is to provide a good outcome, and so the quality of care should be mirrored in the outcome. ${ }^{4}$ Mortality is the most common outcome that has been analyzed because of its clear definition and the ease of availability. There has been a need to create more comprehensive outcomes report cards for $\mathrm{CABG}$ surgery that better reflect institutional quality of care. ${ }^{5}$ Detailed report cards that include outcomes other than mortality have not been widely researched for CABG surgery. ${ }^{6}$

Surveys of cardiologists and cardiac surgeons have been completed to understand the impact of these reports on practice patterns. ${ }^{7,8}$ In Pennsylvania, only $10 \%$ of cardiac surgeons and cardiologists in the state believed that mortality rates were "very important" in assessing surgeon performance, and $87 \%$ of cardiologists stated the guide had a minimal influence or none on their referral patterns. ${ }^{7} \mathrm{~A}$ majority (78\%) of cardiac surgeons and cardiologists believed the most important limitation of these reports was the absence of indicators of quality other than mortality. ${ }^{7}$ In Pennsylvania the CABG surgery guide had limited credibility, and it was concluded that future reports should be collaborative and involve all stakeholders. ${ }^{7}$ It is more likely that reports tailored and targeted to providers will be successful in influencing improvements in the quality of care.

In the United States, peer review organizations monitor hospital use and quality of care in many states with the goals of attempting to reduce patient morbidity and mortality through the identification of preventable mortality and morbidity by implicit chart reviews. These reviews identify outcomes that are related to quality of care and would be preventable if optimal care had been delivered. ${ }^{9}$ This type of review is the most accurate estimate of the proportion of adverse outcomes related to quality of care; however, the use of this process is limited because it is costly and time consuming.

The National Quality Forum (NQF), a private, nonprofit, public benefit corporation whose mission is to improve American health care, recently released a report of voluntary consensus standards for cardiac surgery. ${ }^{10}$ Previous to this there was no consensus set of quality measures or indicators that had been published for CABG surgery. The method of identifying quality indicators in health care through consensus paneling has been successfully applied in many situations including hospital admissions for congestive heart failure and acute myocardial infarction, as well as for emergency visits. ${ }^{11-13}$ This project's goal was to develop a comprehensive set of consensus indicators for $\mathrm{CABG}$ surgery that was believed by an expert panel to measure quality of care, relate to preventable death, and be included as a minimum set on Canadian CABG performance reports.

\section{Methods}

\section{Quality Indicators}

An initial list of potential quality indicators was accumulated through a MEDLINE search, databases of established quality initiatives (eg, Northern New England Cardiovascular Disease Study Group, Society For Thoracic Surgeons), American College of Cardiology/American Heart Association guidelines, and expert opinion. ${ }^{14-19}$ The literature search focused on randomized controlled trial evidence. Quality indicators included variables that were outcomes, surrogate outcomes related to processes of perioperative care, and structure variables. These variables were rated on 5-point Likert scales, and panel members were given an opportunity to suggest quality indicators that were not listed.

\section{Rating Scales}

Outcomes and surrogate outcomes (such as peak troponin level and lowest intraoperative hematocrit) were rated on 4 separate scales. The first scale evaluated the degree to which the implementation of best practice evidence and guidelines could help prevent the outcome $(1=$ extremely preventable, $2=$ very preventable, $3=$ somewhat preventable, $4=$ minimally preventable, $5=$ not at all preventable). The second scale evaluated the adequacy of risk adjustment for this outcome $(1=$ extremely adequate, 2 = very adequate, $3=$ neutral, $4=$ minimally adequate, 5 $=$ not adequate). The third scale evaluated whether the outcome was related to preventable death $(1=$ strongly related, $2=$ very related, $3=$ somewhat related, $4=$ minimally related, $5=$ not at all related). The final scale evaluated the usefulness of the outcome to be used on a surgery report card for institutional quality assur- 
TABLE 1. Consensus Quality Indicators to be reported on coronary artery bypass graft surgery institutional report cards

\begin{tabular}{|c|c|c|c|c|}
\hline \multicolumn{5}{|c|}{ Percentage agreement for various rating scales } \\
\hline Quality indicator & Quality of care & Preventable death & Report card & Risk adjustment \\
\hline \multicolumn{5}{|l|}{ Outcomes } \\
\hline 30-day mortality & $73 \%$ & $87 \%$ & $100 \%$ & $100 \%$ \\
\hline In-hospital mortality & $93 \%$ & $100 \%$ & $100 \%$ & $100 \%$ \\
\hline Postoperative stroke & $67 \%$ & $93 \%$ & $100 \%$ & $93 \%$ \\
\hline Postoperative ECG myocardial infarction & * & $87 \%$ & $80 \%$ & * \\
\hline Deep sternal wound infection & $93 \%$ & $100 \%$ & $100 \%$ & $67 \%$ \\
\hline Postoperative dialysis & * & $80 \%$ & $87 \%$ & $73 \%$ \\
\hline Chest reopening & * & $73 \%$ & $67 \%$ & $*$ \\
\hline ICU readmission & * & * & $67 \%$ & $73 \%$ \\
\hline Ventilation time & * & * & $69 \%$ & * \\
\hline ICU length of stay & $80 \%$ & * & $87 \%$ & $87 \%$ \\
\hline Total packed red blood cells transfused & $73 \%$ & $73 \%$ & $73 \%$ & * \\
\hline Total units of allogeneic blood products & $79 \%$ & $79 \%$ & $79 \%$ & * \\
\hline 365-day repeat operation with CPB & * & $73 \%$ & $73 \%$ & * \\
\hline 365-day repeat revascularization & * & $*$ & $67 \%$ & * \\
\hline \multicolumn{5}{|l|}{ Processes of care } \\
\hline Waiting time to surgery & * & $67 \%$ & $87 \%$ & $\dagger$ \\
\hline Completion of surgery within a recommended waiting time & $67 \%$ & $80 \%$ & $80 \%$ & $\dagger$ \\
\hline Internal thoracic artery to LAD & $100 \%$ & $100 \%$ & $87 \%$ & $\dagger$ \\
\hline \multicolumn{5}{|l|}{ Structure } \\
\hline Institutional volume & * & * & $67 \%$ & $\dagger$ \\
\hline
\end{tabular}

ECG, Electrocardiogram; ICU, intensive care unit; $C P B$, cardiopulmonary bypass; $L A D$, left anterior descending. *Less than two thirds reached a consensus on this scale. $†$ Rating scale not applicable to this variable.

ance $(1=$ extremely useful, $2=$ very useful, $3=$ somewhat useful, $4=$ minimally useful, $5=$ not at all useful).

The process of care variables and structure variables were rated on 3 separate scales. The first scale evaluated whether the process or structure variable was related to preventing adverse outcomes after CABG surgery $(1=$ strongly related, $2=$ very related, $3=$ somewhat related, $4=$ minimally related, $5=$ not at all related). The second scale evaluated whether the process or structure variable was related to preventable death $(1=$ strongly related, $2=$ very related, $3=$ somewhat related, $4=$ minimally related, $5=$ not at all related). The third scale evaluated how useful it would be to measure process or structure variables on CABG surgery report cards for institutional quality assurance $(1=$ extremely useful, $2=$ very useful, $3=$ somewhat useful, $4=$ minimally useful, $5=$ not at all useful).

\section{Delphi Consensus Panel}

Delphi consensus paneling is a process that involves consulting a group of experts on an issue. ${ }^{20}$ Through multiple ratings with anonymous feedback between rounds of ratings (including providing their response to questions in relation to the panel's response), a clear consensus on the issue is reached. ${ }^{20}$ Our panel consisted of 19 members who were multidisciplinary, including cardiac surgeons from 10 hospitals in Ontario and Quebec, 2 cardiac anesthetists, 1 US surgeon (Frederick Grover) involved in quality initiatives of the Society for Thoracic Surgery and Veteran's Affairs system, 1 outcomes researcher from Alberta, and a quality of care researcher (Gerald T. O'Connor) heading quality initiatives in the Northern New England region. Nominations for members of the panel were requested from the Heart and Stroke Foundation of Canada and the Canadian Cardiovascular Society. The panel rated all potential quality indicators during the first round through an e-mail survey, and a second rating was completed after each variable was discussed at a full day meeting. Those variables on which two thirds of the panel reached a consensus that there was a relation to quality of care, relation to preventable death, and/or need for inclusion on a surgical report card were developed further. The definitions of the chosen quality indicators were then discussed and clarified by the panel through a series of four 1-hour teleconferences. The quality indicators derived from our panel were also compared with the list derived from the NQF's voluntary standards consensus report on cardiac surgery.

\section{Results}

This report includes a summary of the results of the consensus panel discussion of quality measures. Detailed indicator definitions are available at http://www.qualitycabg.org for readers who would like to use them for CABG surgery databases or CABG surgery institutional performance profiling. The quality measures that were evaluated in the consensus process were classified into major categories including outcomes, processes of care, and structure variables. Preventable death was considered the most important quality measure, and all other potential quality indicators were rated regarding their relation to this important variable. A total of 149 potential quality indicators were evaluated in 
TABLE 2. Comparison of Canadian Consensus Quality Indicators for standardized reporting in relation to

\begin{tabular}{|c|c|c|}
\hline \multicolumn{3}{|c|}{ National Quality Forum Voluntary Consensus Standards for cardiac surgery } \\
\hline Quality indicator & Canadian Consensus Panel & NOF Consensus Standards \\
\hline \multicolumn{3}{|l|}{ Structure } \\
\hline Participation in a cardiac surgery database & Encouraged & Encouraged \\
\hline Case volume & Institutional volume & $\begin{array}{l}\text { Volume for CABG, valve, and CABG + valve } \\
\text { surgery }\end{array}$ \\
\hline \multicolumn{3}{|l|}{ Processes of care } \\
\hline Timing of antibiotic administration & Not included & Included \\
\hline Selection of antibiotic administration & Not included & Included \\
\hline Duration of antibiotic prophylaxis & Not included & Included \\
\hline Preoperative beta-blockade & Not included & Included \\
\hline Internal thoracic artery use & If bypassed to LAD artery & Included \\
\hline Waiting time to surgery & Included & Not included \\
\hline $\begin{array}{l}\text { Completion of surgery within a } \\
\text { recommendedwaiting time according to } \\
\text { patient's symptoms }\end{array}$ & Included & Not included \\
\hline Antiplatelet medications at discharge & Not included & Included \\
\hline Beta-blockade at discharge & Not included & Included \\
\hline Anti-lipid treatment at discharge & Not included & Included \\
\hline \multicolumn{3}{|l|}{ Outcomes } \\
\hline 30-day mortality & Included & $\begin{array}{l}\text { Included but also indicates all in-hospital deaths } \\
\text { regardless of timing }\end{array}$ \\
\hline In-hospital mortality & Included & Included \\
\hline Operative mortality (AVR) & Valve surgery not included in project & Included \\
\hline Operative mortality (MVR) & Valve surgery not included in project & Included \\
\hline Operative mortality (MVR+CABG) & Valve surgery not included in project & Included \\
\hline Operative mortality (AVR + CABG) & Valve surgery not included in project & Included \\
\hline Ventilation time & Included & Included as prolonged intubation \\
\hline ICU length of stay & Included & Not included \\
\hline Chest reopening & Included & Included as surgical reexploration \\
\hline ICU readmission & Included & Not included \\
\hline Postoperative stroke & Included & Included \\
\hline Postoperative ECG myocardial infarction & Included & Not included \\
\hline Deep sternal wound infection & Included & Included \\
\hline Postoperative dialysis & Included & Included as postoperative renal insufficiency \\
\hline Total packed red blood cells transfused & Included & Not included \\
\hline Total units of blood products transfused & Included & Not included \\
\hline 365-day repeat operation with CPB & Included & Not included \\
\hline 365-day repeat revascularization & Included & Not included \\
\hline
\end{tabular}

$N Q F$, National Quality Forum; $A V R$, aortic valve replacement; $M V R$, mitral valve replacement; $C A B G$, coronary artery bypass graft; ICU, intensive care unit; $L A D$, left anterior descending; $E C G$, electrocardiogram; $C P B$, cardiopulmonary bypass.

this Delphi process including 54 outcome variables, 20 surrogate outcome variables, 72 process of care variables, and 3 structure variables.

A total of 18 variables were agreed on as important enough to be included on an institutional performance report for CABG surgery (Table 1). This included 14 outcome variables, 3 process of care variables, and 1 structure variable (Table 1). It was interesting to note that both 30-day mortality, which takes into account out-of-hospital mortality at a specific time-point, and in-hospital mortality were considered important as separate measures of quality (Table 1). These measures were rated highly for all rating scales and have in the past been used as standard variables on CABG report cards (Table 1).

For measures such as ventilation time, intensive care unit (ICU) readmission, 365-day repeat revascularization, and institutional volume, there was a consensus regarding inclusion in an institutional performance report; however, there was no consensus on their relation to quality of care and preventable death (Table 1). There was no consensus whether robust risk-adjustment was available for some outcomes that were otherwise believed by the panel to be important enough to include on report cards, including postoperative electrocardiographic (ECG) myocardial infarction, ventilation 
TABLE 3. Coronary artery bypass graft surgery quality indicators rated highly for quality of care and/or preventable death on which no consensus was reached for use in an institutional performance report

\begin{tabular}{|c|c|c|c|}
\hline \multicolumn{4}{|c|}{ Percentage agreement for various rating scales } \\
\hline Quality indicator & Quality of care & Preventable death & Risk Adjustment \\
\hline \multicolumn{4}{|l|}{ Outcomes } \\
\hline Postoperative length of stay & $67 \%$ & * & $73 \%$ \\
\hline New postoperative IABP & * & $67 \%$ & $67 \%$ \\
\hline Inotropic support required at 24 and $48 \mathrm{~h}$ & * & $80 \%$ & $80 \%$ \\
\hline Postoperative cardiac arrest & * & $80 \%$ & * \\
\hline Reintubation rate & $92 \%$ & $86 \%$ & * \\
\hline \multicolumn{4}{|l|}{ Processes of care } \\
\hline IV nitroglycerin until time of OR for CCS IV angina & $67 \%$ & * & $\dagger$ \\
\hline Continue IV heparin or LMWH until OR for recent ACS & * & $67 \%$ & $\dagger$ \\
\hline Stop ADP inhibitors preoperatively & $67 \%$ & * & $\dagger$ \\
\hline Continue ASA until OR for elective patients & $73 \%$ & $67 \%$ & $\dagger$ \\
\hline Timely administration of preoperative prophylactic antibiotics & $93 \%$ & $70 \%$ & $\dagger$ \\
\hline Administration of antifibrinolytics for reoperative CABG surgery & $80 \%$ & * & $\dagger$ \\
\hline TEE at the beginning and end of every case & $73 \%$ & $73 \%$ & $\dagger$ \\
\hline Assessment of aorta by epiaortic ultrasound & $80 \%$ & $73 \%$ & $\dagger$ \\
\hline Complete revascularization & $87 \%$ & $80 \%$ & $\dagger$ \\
\hline Assessment of graft patency at the end of every case & $93 \%$ & $87 \%$ & $\dagger$ \\
\hline Myocardial protection with blood cardioplegia & $80 \%$ & $73 \%$ & $\dagger$ \\
\hline Initiation of ASA or other antiplatelet within $24 \mathrm{~h}$ postoperatively & $87 \%$ & $73 \%$ & $\dagger$ \\
\hline Insulin infusion initiated within $24 \mathrm{~h}$ for elevated glucose level & $87 \%$ & $73 \%$ & $\dagger$ \\
\hline Initiation of beta-blocker & $67 \%$ & * & $\dagger$ \\
\hline Continuous telemetry monitoring on the ward & $67 \%$ & * & $\dagger$ \\
\hline Postoperative ASA administered & $80 \%$ & $73 \%$ & $\dagger$ \\
\hline Anticoagulation with heparin for $48 \mathrm{~h}$ of atrial fibrillation & $87 \%$ & $80 \%$ & $\dagger$ \\
\hline Postoperative beta-blocker administered & $93 \%$ & $87 \%$ & $\dagger$ \\
\hline Postoperative antihyperlipidemic administered & $87 \%$ & $91 \%$ & $\dagger$ \\
\hline Postoperative ACE inhibitor administered & $87 \%$ & $91 \%$ & $\dagger$ \\
\hline \multicolumn{4}{|l|}{ Structure } \\
\hline Nurse to patient ratio in the ICU & $87 \%$ & $80 \%$ & $\dagger$ \\
\hline Nurse to patient ratio on the ward & $87 \%$ & $80 \%$ & $\dagger$ \\
\hline Daily ward care managed by dedicated NP or MD & $67 \%$ & * & $\dagger$ \\
\hline
\end{tabular}

$\overline{I A B P}$, Intra-aortic balloon pump; $O R$, operating room; CCS, Canadian Cardiovascular Society angina class; $I V$, intravenous; $L M W H$, low molecular weight heparin; $A D P$, adenosine diphosphate platelet receptor; $A S A$, acetylsalicylic acid; $T E E$, transesophageal echocardiogram; $A C E$, angiotensin-converting enzyme; $I C U$, intensive care unit; $N P$, nurse practitioner; $A C S$, acute coronary syndrome; $C A B G$, coronary artery bypass graft. *Less than two thirds reached a consensus on this scale. †Rating scale not applicable to this variable.

time, total packed cells transfused, total units of blood products transfused, ICU length of stay, chest reopening, 365-day repeat operation with cardiopulmonary bypass, and 365-day repeat revascularization (Table 1).

The process of care variables that it was agreed should be reported included waiting time to surgery and completion of surgery within a recommended waiting time depending on the triage urgency of a case (Table 1). These measures are important in the Canadian public health care system in which resource constraints lead to waits for invasive procedures. The only other process of care that was agreed should be reported was the use of the internal thoracic artery in left anterior descending bypass surgery (Table 1).

The variables selected by our panel as appropriate for reporting on institutional report cards were further com- pared with the NQF's consensus standards for cardiac surgery list (Table 2). It is interesting to note that $50 \%$ of the quality indicators selected for reporting by our panel overlapped with the NQF's voluntary standards (Table 2). The NQF's set included a number of process variables that were not selected by our panel (despite being reviewed during the Delphi process), including the type and timing of preoperative antibiotics, the perioperative use of beta blockade, and the postoperative use of antiplatelet agents and lipidlowering agents (Table 2). The NQF's set of standards included valve-related outcomes, which were not in the scope of our project (Table 2). Conversely the NQF's standards did not include variables unique to a public health care system including waiting time to surgery and surgery within a recommended waiting time based on a patient's symptomatic 
status. There were a number of outcome variables that the Canadian panel included as important in measuring institutional performance that the NQF's set did not include, such as 1-year repeat revascularization, perioperative rates of allogeneic blood transfusion, postoperative ECG myocardial infarction, and ICU readmissions and length of stay.

There were variables that despite being labeled by the panel as related to quality of care, preventable death, or both were not included in those chosen for institutional reporting. Some of these were process of care variables that the NQF included in their set (Table 3). It was interesting that hospital length of stay was one of these variables, despite the fact it had been used as a standard measure for CABG surgery reports in Ontario in the past.

There were a number of variables that by consensus were believed to be related both to quality of care and preventable death but were not believed to be useful on an institutional report card (Table 3). Many of these were process and structure variables in which the requirement of technology or staffing was not monetarily feasible within a publicly funded hospital budget, such as the use of transesophageal echocardiograms at the beginning and end of each case, assessment of the aorta by epiaortic ultrasound, intraoperative assessment of graft patency, nurse to patient ratio in the ICU, and nurse to patient ratio on the ward. These variables were believed to be important to measure to understand where it may be necessary to lobby for funding; however, the panel agreed reporting at an institutional level would only be reflective of the public funding available to a particular surgical service, and not necessarily the performance.

Other variables (Table 3) that were believed to be related both to quality of care and preventable death were mainly medication administration processes of care, including the use of preoperative aspirin until the day of surgery for elective patients, timely administration of prophylactic preoperative antibiotics to prevent wound infection, initiation of aspirin or other antiplatelet within 24 hours after surgery, insulin infusion initiation within 24 hours of surgery for elevated glucose, postoperative aspirin use, anticoagulation for patients with episodes of atrial fibrillation that continue over a 48-hour period, postoperative beta-blocker use, postoperative antihyperlipidemic use, and postoperative angiotensinconverting enzyme inhibitor use.

The intraoperative variables that were believed to be important both for preventable death and quality of care included complete revascularization and blood cardioplegia.

\section{Discussion}

We hope this set of quality indicators for CABG surgery will serve as a feasible, standard list that will be monitored by providers of CABG surgery in an effort to continuously evaluate and improve their performance in Canada. After the evaluation of an extensive list of potential indicators we were able to narrow down the variables to measures that were agreed as most important to collect and report. Many of the variables included in this set can be derived from existing clinical and administrative databases, an important consideration in a public health care system with limited funding available for such activities. In addition, we hope to abstract those variables that are not currently available in any database on an ongoing basis through chart abstraction in a regional initiative to improve quality of care in Ontario.

The set of quality indicators that we developed include a broad range of all aspects of the Donabedian model of health care including outcomes, process, and structure variables related to CABG surgery. We have distinctly defined which indicators the majority of an expert panel agree relate to the concrete concept of the Institute of Medicine's definition of quality of care and another important measure of quality, namely, preventable death. ${ }^{21-23}$ The panel also took into consideration that this quality indicator set was to be applied in the unique setting of public health care, where resource constraints lead to queuing for procedures and efficiency is a crucial quality measure in allowing surgical services to move patients through the system.

The primary intent in developing this quality indicator set was to encourage providers to voluntarily collect and report their outcomes as has occurred for basic outcomes in the province of Ontario. We anticipate with limited capacity for cardiac surgical services and little consumer choice, quality initiatives will serve to provide public accountability and encourage provider quality improvement. However, because of a lack of excess services it is unlikely that consumers will shift to higher performing providers in Canada. In our country there have been no mandatory outcomes reporting initiatives, and public reporting only exists at an institutional level in one province (ie, Ontario). Because quality improvement initiatives in Canada are voluntary, there has been no pressure to report outcomes at a surgeon level.

Our panel was instructed to select an ideal set of measures that could be used to assess quality of care, were feasible to collect, and could be included in yearly institutional cardiac surgery report cards. Despite the fact our clinicians believed that process measures, such as those selected in the NQF process, were related to quality of care and preventable death, they ultimately prioritized a set of measures that included mainly outcome measures. Our panel thought outcomes feedback would be most useful to providers in measuring institutional performance. Our panel further thought that in the absence of clear randomized trial evidence for the timing, and perioperative use of many process measures, it would not provide the most impact in guiding providers toward a need for quality improvement. 
Despite this, we acknowledge there is substantial evidence to support the process measures contained in the NQF set, and these process measures are widely used by many clinicians.

The Delphi consensus expert panel process involving both providers and health care research methodologists was used to develop these indicators to ensure their clinical relevancy and importance in relating to quality of care. ${ }^{13} \mathrm{It}$ has been suggested and shown that providers of CABG surgery, where established public reporting has existed for years, are ambivalent to these reports, and this may be because of limited stakeholder involvement in the process of measuring and reporting quality of care. Regions such as New York State and Pennsylvania are examples where governmental decree has led to the production of CABG surgery reports that contain quality measures limited to mortality, length of stay, and readmission to hospital. ${ }^{7,8}$ The perspective of our panel was mainly to target quality improvement in providers through feedback, rather than shifting consumers to those providers with better outcomes. It is interesting to note that the NQF panel included purchasers, consumers, and payors in addition to providers, which could explain the difference in the composition of their set of quality measures. The NQF panel stipulated that the measures to be included in their set must already be widely accepted and in use by providers.

The measurement and reporting of quality indicators in CABG surgery has been identified as vital in improving quality of care as reflected by improvements observed in regions where this has already been implemented. ${ }^{14,15,24-27} \mathrm{We}$ recognize the set of quality measures selected in this study will require update with evolving evidence and may need to be modified because of regional differences in the delivery of CABG care. Despite this caveat, it is reassuring to observe there was a significant degree of overlap in the quality indicators selected in our study in comparison with the NQF process of voluntary consensus standards for cardiac surgery in the United States.

The derivation of quality indicators in our study was in the context of CABG surgery in a public health care system, such as Canada. To this endeavor our set of quality indicators included "waiting time to surgery" and "completion of surgery within a maximum recommended waiting time," measures that would not be an issue in most regions of the United States. These measures were included to monitor for deficiencies in the capacity of our health care system (ie, not an institutional problem but a governmental health care funding issue) and to ensure that all institutions efficiently direct patients through the queue for surgery, including transferring patients to other institutions to receive surgery in a timely fashion. All other measures were defined in a way to ensure they were consistent with existing databases including The Society for Thoracic Surgery database.
Other unique aspects of our set include our ability to track patients out to 1 year after surgery using administrative databases available in the Canadian public health care system. Thus, our panel decided to include measures such as "365-day repeat surgery requiring cardiopulmonary bypass" and "repeat revascularization." These measures span beyond the classic in-hospital outcomes and measure the durability of a surgical treatment. Such measures can be influenced by a variety of factors including the technical quality of the surgery, effectiveness of the procedure including the choice of patient, and quality of postoperative care. In the United States, such measures may also be of interest to payors and purchasers of health care in assessing the relative value of a treatment choice, especially because currently the evidence is not clear on whether multivessel angioplasty may be superior to CABG. In Canada, provincial governments are in control of healthcare dollars and frequently use observational data to plan healthcare funding, including the relative distribution of funds to various hospital programs.

The challenge with many of our outcome quality measures is that robust risk adjustment is not currently available. This was recognized by the panel and will require further investigation. A further consideration is the ease of interpretation of the quality measure. The NQF set of measures included a larger proportion of process measures that are easier to define and report, and do not require risk adjustment other than the exclusion of ineligible patients. On the other hand, outcome measures require risk adjustment and may be harder to translate to the lay public. Because our set mainly includes outcomes, we will have to provide a translational document to assist with the interpretation of such data by more general audiences. We further hope to be able to provide the relationships between the different categories of quality indicators, and thus a more accurate snapshot of quality at an institutional level.

A limitation of our study was that during the panel process members may have been biased in choosing certain indicators for inclusion. However, we suspect these biases would be minimized because of the multidisciplinary nature of the panel (ie, a diverse range of participants including providers, Canadian and US members, and health policy methodologists). During the panel process, the panel was allowed to freely suggest, discuss, and add further quality measures to the initial list.

\section{Conclusion}

We used a formal process to identify and define quality indicators that are most important to monitor quality of care for CABG surgery in a continuous quality improvement effort. We hope this set of indicators will be a useful starting point for individual surgeons or hospitals as well as regions interested in embarking on initiating a continuous quality 
improvement initiative through measurement and feedback for CABG surgery. In our region we will be testing the feasibility of measuring these indicators and hope to eventually set indicator benchmarks regarding performance that our hospitals can aim to strive toward.

\section{Author Appendix}

The Canadian CABG Surgery Quality Indicator Consensus Panel consisted of Sewa Aul (Chief of Cardiac Surgery, Sudbury Regional Hospital), Gopal Bhatnagar (Chief of Cardiac Surgery, Trillium Health Centre), Michael Bourke (Staff Intensivist/Anesthetist, Ottawa Heart Institute), Kathy Deemar (Perfusionist, Sunnybrook and Women's College Health Sciences Centre), William A. Ghali (Professor, Faculty of Medicine, University of Alberta), Christopher M. Feindel (Staff Cardiac Surgeon, University Health Network), Andrew Hamilton (Chief of Cardiac Surgery, Kingston General Hospital), Andre Lamy (Staff Cardiac Surgeon, Hamilton Health Sciences Centre), David Latter (Staff Cardiac Surgeon, St Michael's Hospital), Jean Francois Morin (Staff Cardiac Surgeon, Jewish General Hospital, Montreal, Quebec), Patricia Murphy (Staff Intensivist/Anesthetist, University Health Network), Richard J. Novick (Chief of Cardiac Surgery, London Health Sciences Centre), and Fraser Rubens (Staff Cardiac Surgeon, Ottawa Heart Institute).

We thank Sharon Vanihinger, for administrative support in the organization of the consensus panel, and Jeri Sever, for database creation and data entry.

\section{References}

1. Nallamothu BK, Saint S, Ramsey SD, et al. The role of hospital volume in coronary artery bypass grafting: is more always better? $J \mathrm{Am}$ Coll Cardiol. 2001;38(7):1923-30.

2. Shahian DM, Williamson WA, Svensson LG, et al. Applications of statistical quality control to cardiac surgery. Ann Thorac Surg. 1996; 62(5):1351-8; discussion 1358-9.

3. Kohn LT, Corrigan JM, Donaldson MS. To err is human. Washington: National Academy Press; 2000.

4. Hartz AJ, Kuhn EM. Comparing hospitals that perform coronary artery bypass surgery: the effect of outcome measures and data sources. Am J Public Health. 1994;84(10):1609-14.

5. O'Brien MM, Shroyer AL, Moritz TE, et al. Relationship between processes of care and coronary bypass operative mortality and morbidity. Med Care. 2004;42(1):59-70.

6. Fortescue EB, Kahn K, Bates DW. Prediction rules for complications in coronary bypass surgery: a comparison and methodological critique. Med Care. 2000;38(8):820-35.

7. Schneider EC, Epstein AM. Influence of cardiac-surgery performance reports on referral practices and access to care. A survey of cardiovascular specialists. $N$ Engl J Med. 1996;335(4):251-6.

8. Hannan EL, Stone CC, Biddle TL, DeBuono BA. Public release of cardiac surgery outcomes data in New York: what do New York state cardiologists think of it? Am Heart J. 1997;134(1):55-61.

9. Hannan EL, Kilburn H, Jr., O'Donnell JF, et al. Adult open heart surgery in New York State. An analysis of risk factors and hospital mortality rates. JAMA. 1990;264(21):2768-74.
10. National Quality Forum. National voluntary consensus standards for cardiac surgery (National Quality Forum). Washington, DC: National Quality Forum; 2004, www.qualityforum.org.

11. Lee DS, Tran C, Flintoft V, et al. CCORT/CCS quality indicators for congestive heart failure care. Can J Cardiol. 2003;19(4):357-64.

12. Tran CT, Lee DS, Flintoft VF, et al. CCORT/CCS quality indicators for acute myocardial infarction care. Can J Cardiol. 2003;19(1):38-45.

13. Lindsay P, Schull M, Bronskill S, Anderson G. The development of indicators to measure the quality of clinical care in emergency departments following a modified-Delphi approach. Acad Emerg Med. 2002; 9(11):1131-9.

14. O'Connor GT, Plume SK, Olmstead EM, et al. A regional intervention to improve the hospital mortality associated with coronary artery bypass graft surgery. The Northern New England Cardiovascular Disease Study Group. JAMA. 1996;275(11):841-6.

15. Grover FL, Shroyer AL, Hammermeister K, et al. A decade's experience with quality improvement in cardiac surgery using the Veterans Affairs and Society of Thoracic Surgeons national databases. Ann Surg. 2001;234(4):464-72; discussion 472-4.

16. Eagle KA, Guyton RA, Davidoff R, et al. ACC/AHA guidelines for coronary artery bypass graft surgery: executive summary and recommendations: A report of the American College of Cardiology/American Heart Association Task Force on Practice Guidelines (Committee to revise the 1991 guidelines for coronary artery bypass graft surgery). Circulation. 1999;100(13):1464-80.

17. Eagle KA, Guyton RA, Davidoff R, et al. ACC/AHA Guidelines for Coronary Artery Bypass Graft Surgery: A Report of the American College of Cardiology/American Heart Association Task Force on Practice Guidelines (Committee to Revise the 1991 Guidelines for Coronary Artery Bypass Graft Surgery). American College of Cardiology/American Heart Association. J Am Coll Cardiol. 1999;34(4): 1262-347.

18. Shroyer AL, London MJ, Sethi GK, et al. Relationships between patient-related risk factors, processes, structures, and outcomes of cardiac surgical care. Conceptual models. Med Care. 1995;33(10 Suppl):OS26-34/.

19. Shroyer AL, London MJ, VillaNueva CB, et al. The Processes, Structures, and Outcomes of Care in Cardiac Surgery study protocol. Med Care. 1995;33(10 Suppl):OS17-25.

20. Jones J, Hunter D. Consensus methods for medical and health services research. BMJ. 1995;311(7001):376-80.

21. Dubois RW, Brook RH. Preventable deaths: who, how often, and why? Ann Intern Med. 1988;109(7):582-9.

22. Brennan TA, Leape LL, Laird NM, et al. Incidence of adverse events and negligence in hospitalized patients. Results of the Harvard Medical Practice Study I. N Engl J Med. 1991;324(6):370-6.

23. Gawande AA, Thomas EJ, Zinner MJ, Brennan TA. The incidence and nature of surgical adverse events in Colorado and Utah in 1992. Surgery. 1999;126(1):66-75.

24. Hannan EL, Kilburn H, Jr., Racz M, et al. Improving the outcomes of coronary artery bypass surgery in New York State. JAMA. 1994; 271(10):761-6.

25. Hammermeister KE, Johnson R, Marshall G, Grover FL. Continuous assessment and improvement in quality of care. A model from the Department of Veterans Affairs Cardiac Surgery. Ann Surg. 1994; 219(3):281-90.

26. Ferguson TB, Jr., Peterson ED, Coombs LP, et al. Use of continuous quality improvement to increase use of process measures in patients undergoing coronary artery bypass graft surgery: a randomized controlled trial. JAMA. 2003;290(1):49-56.

27. Hannan EL, Kumar D, Racz M, et al. New York State's Cardiac Surgery Reporting System: four years later. Ann Thorac Surg. 1994;58(6):1852-7. 


\section{Electronic Appendix: Canadian Quality Indicator Project (Funded by a Research Grant From the Heart and Stroke Foundation of Ontario HSF 5484) Appendix of Definitions ${ }^{\dagger}$}

*Those indicators recommended by the consensus panel members to be collected and included in CABG surgery performance reports for the purpose of quality improvement. The remaining indicators were rated by the panel as significantly related to quality of care and/or preventable death following CABG surgery

\section{Outcomes}

*30-day mortality: Any death occurring within 30 days following the operative date.

*In-hospital mortality: A death occurring at any point during the hospital stay.

Hospital postoperative length of stay: The total number of days spent in hospital from the date of surgery to the date of discharge home, or transfer to another acute care facility/long-term care facility. The variables to be collected include: date of surgery, date of discharge, and discharged to either (1) home, (2) acute care facility, (3) long term care facility (this information must be chart abstracted).

\section{The following outcome is for measurement during the} intraoperative time period only:

Reinstitution of CPB after initial separation: The requirement to put a patient back on cardiopulmonary bypass as indicated by the perfusion records through the following flags: administration of protamine, full reheparinization, and reinitiation of cardiopulmonary bypass.

\section{The following outcomes are for measurement during the in-hospital time period only:}

New postoperative intra-aortic balloon support: The insertion of an intra-aortic balloon pump at any point after grafting the heart including intraoperatively and in the intensive care unit (administrative data will only indicate if the balloon was inserted on the day of surgery or after this day).

Inotropic support requirement at 24 and 48 hours: The postoperative usage of two or more inotropes at 24 and 48 hours after the operation in doses to maintain adequate hemodynamic support (systolic bp $>90 \mathrm{~mm} \mathrm{Hg}$, C.I. $>2.2$, U.O. $>0.5$ $\mathrm{cc} / \mathrm{kg} / \mathrm{hr}$ ). The names and doses of the inotrope infusions will be abstracted as well as the: cardiac index, cardiac output, weight, height, average urine output over the last 8 hours, and blood pressure (systolic/diastolic) for these time points (eg, dobutamine, dopamine, epinephrine, phenylephrine, norepinephrine, vasopressin, milrinone)

*Postoperative in-hospital myocardial infarction ECG criteria: This will measure an ECG established myocardial infarction (must be a change from the initial preoperative ECG, excluding those patients with a preoperative bundle branch block or paced rhythm). Established myocardial infarction is defined as either (1) new onset left bundle branch block, (2) loss of $\mathrm{R}$ waves in the anterior leads, (3) any Q wave in leads V1 through V3, Q wave $\geq$ to $30 \mathrm{~ms}(0.03 \mathrm{~s})$ in leads I, II, aVL, aVF, V4, V5 or V6. (The Q

$\dagger$ Available on Web site at http://www.qualitycabg.org. wave changes must be present in any two contiguous leads, and be $\geq 1 \mathrm{~mm}$ in depth.) A preoperative ECG will be compared to the latest ECG before discharge from hospital (the date of each ECG will be included in this variable).

Postoperative cardiac arrest on the ward: An incident when a patient is on the ward, where the arrest team is called (either for a respiratory arrest or rhythm with inadequate blood pressure) resulting in the need of defibrillation, chest compressions and/or ventilatory support.

*Ventilation time: The number of hours during which the patient has an endotracheal tube or tracheostomy with ventilation (ie includes from the time noted at the end of skin closure to the time of removal of the endotracheal tube from the patient and any reintubation ventilation times).

Reintubation rate: The requirement for a patient to be reintubated after initial extubation following surgery at any point during the hospital stay.

*Total units of packed red blood cells transfused: The total number of units of packed red blood cells transfused intraoperatively and postoperatively during the hospital stay. Administrative data can only capture if red cells were transfused during the admission.

*Total units of allogeneic blood products transfused: The total number of units of allogeneic blood products (other than packed red blood cells) transfused intraoperatively and postoperatively during the hospital stay. Administrative data can only capture if any allogeneic blood products were transfused.

*Deep sternal wound infection including mediastinitis/osteomyelitis: A sternal wound infection involving the deep soft tissues (eg, fascial, muscle, bone and/or mediastinum) with any one of the following conditions: (1) purulent drainage from deep incision; (2) wound opened either spontaneously or by a surgeon with the patient having one of the following signs or symptoms: fever $>38^{\circ} \mathrm{C}$, or localized pain/tenderness, unless incision is culture negative; (3) an abscess or other evidence of infection involving the deep incision is found on direct examination, during reoperation or by histopathologic or radiologic examination; (4) diagnosis of deep incisional surgical site infection by a surgeon or attending physician.

*New postoperative stroke: A central neurologic deficit persisting postoperatively for $>72$ hours with evidence on an imaging study (ie CT scan, MRI) and/or neurologist confirmation.

*New requirement for postoperative dialysis: A new postoperative requirement for hemodialysis (including any new renal replacement therapy-CRRT, CVVHD) at any time during the hospital stay (ie, patients are excluded if they have had preoperative dialysis or isolated intraoperative dialysis).

*ICU readmission: The requirement of a patient to be transferred to the intensive care unit after initial discharge to the ward (not including step down units, including date of readmission and date of discharge).

*Intensive care unit length of stay: The total number of days spent in the intensive care unit after surgery (not including step down unit stays) including readmission stays to the ICU (include date of initial discharge from ICU).

*Chest reopening for any reason: Requirement of chest reopening after surgery (ie after the patient has left the OR) for any reason (the reason will be abstracted: [1] suspected intrathoracic 
hemorrhage, [2] cardiac tamponade, [3] graft occlusion, [4] arrest, [5] low output syndrome, [6] sternal rewiring/debridement, [7] other:reason to be listed) during a patient's hospital stay.

\section{The following outcomes are for measurement in those} patients that are discharged alive:

*365-day repeat cardiac operation requiring cardiopulmonary bypass: A reoperation within 365 days of the surgery post discharge identified through billing codes for cardiopulmonary bypass, and/or repeat coronary bypass operation.

*365-day repeat revascularization-angioplasty: An angioplasty procedure within 365 days of the surgery (post discharge) identified through the billing code for angioplasty.

365-day myocardial infarction: Applicable to those patients discharged alive and readmitted to hospital as identified from ICD-9 or ICD-10 codes for myocardial infarction.

365-day readmission for cardiac diagnosis (MI, UA, CHF): A readmission to hospital for the primary diagnosis of myocardial infarction, unstable angina or congestive heart failure within 365 days of the surgery post discharge identified through ICD-9 or ICD-10 codes.

30-day all cause readmission rate: Applicable to those patients discharged alive and readmitted to hospital as identified from ICD-9 or ICD-10 codes.

Intra-aortic balloon pump (ie severe LV dysfunction/shock/ persisting anginal symptoms): The preoperative insertion of an intra-aortic balloon pump before grafting or precardiopulmonary bypass for any reason (the reason for insertion will be recorded: hemodynamic instability, severe left ventricular dysfunction (EF $<20 \%$ ) and/or unstable angina not alleviated by maximal medical therapy).

IV nitroglycerin until time of surgery if patient has CCS Class IV angina (if bp permits): The continuation of nitrogylcerin (IV, PO, TD) in those patients having angina at rest with maximal medical therapy and an adequate blood pressure without a requirement for inotropic or intra-aortic balloon support.

Continue IV heparin/LMWH until time of OR for those with recent ACS (eg MI, UA): The continuation of intravenous heparin or low molecular weight heparin (ie, dalteparin should be stopped 12 hours in advance, enoxaparin should be stopped 24 hours in advance) until the time of OR in those patients who are having surgery during the same hospital stay as an admission for an acute coronary syndrome.

Stop ADP inhbitors: The stoppage for urgent and elective patients of: ADP inhibitors (adenosine diphosphate inhibitors eg ticlopidine) at time of the decision for urgent surgery (if $<5$ days from planned date of surgery) or $\geq 5$ days before planned elective surgery date (the preoperative use of Plavix and the date that Plavix is stopped will be abstracted).

Continue ASA until day of surgery for elective patients: The continuation of aspirin (ASA) until the day of surgery in those patients who are having elective surgery (this excludes those patients that have an absolute contraindication to aspirin; ie, allergy, gastic ulcer, or other physician-stated reason). The preoperative usage of aspirin will be recorded as well as the date that aspirin was stopped by the patient.

\section{Processes of Care}

*Waiting time to surgery (ie, OR date from time of referral to surgery): The number of days between the surgical referral date, surgical acceptance date, and the operative date.

*Facility responsiveness (CABG completed within recommended waiting time): If the surgery was completed within the Cardiac Care Network (CCN) of Ontario's recommended waiting time, based on the relative urgency of the patient's clinical status.

Timely administration of wound infection prophylactic antibiotics (within 30 minutes of incision and again if the $O R$ exceeds 3-4 hours): The administration of the appropriate antibiotic choice (ie, cephalosporin or vancomycin for penicillin allergy) within 30 minutes of the skin incision for every patient undergoing CABG surgery (document the time antibiotics given intraoperatively in relation to skin incision time-a maximum of two doses will be recorded, for those who are penicillin allergic and receive vancomycin a second dose will not be required).

Administration of antifibrinolytics for reoperative CABG surgery: The administration of aprotinin or transexamic acid for patients undergoing reoperative CABG surgery to minimize blood loss.

TEE at beginning and end of case for every patient: The use of intraoperative transesophageal echocardiography to monitor ventricular function, aortic disease, and mitral valve competency at the beginning and end of the case.

Assessment of aorta (ie, by epiaortic ultrasound scanning): The assessment of the aorta before cannulation to avoid atherosclerotic \pm calcified plaques by epiaortic ultrasound scanning.

Internal mammary artery to LAD if no absolute contraindication: The use of an internal mammary artery (the type of grafts either right or left, in-situ or free will be recorded) to bypass the territory supplied by the left anterior descending coronary artery in a patient with no absolute contraindications where the IMA is unavailable (the reason for it being unavailable will be recorded including the options of injury and previous usage at primary $\mathrm{CABG})$.

Complete revascularization: The grafting of all major territories of coronary artery circulation where there is a significant stenosis present (the assessment includes: coronary dominance, anatomy of significant stenoses defined as those $\geq 70 \%$ except for left main where a significant stenosis is $\geq 50 \%$ ).

Assessment of graft patency/flow: The assessment of the flow or patency of grafts intraoperatively using either Doppler study, TEE, or intraoperative angiography.

Myocardial protection with blood cardioplegia: The use of blood cardioplegia to provide optimal myocardial protection during crossclamping for those patients undergoing on-pump CABG.

CPB time: The time in minutes spent on cardiopulmonary bypass during the initial surgery.

Initiation of aspirin (or other antiplatelet) within 24 hours following surgery: The use of aspirin or ADP inhibitor (if ASA not tolerated) within 24 hours following bypass surgery involving vein grafts (ie, the time and date of the first dose of postoperative ASA will be recorded).

Insulin infusion postop (for diagnosed and undiagnosed diabetics) within 24 hours of surgery: The initiation of an insulin infusion in the ICU for those patients with a random blood glucose of $>11.1 \mathrm{mmol} / \mathrm{L}$ within 24 hours following surgery (ie, the time 
and date of the initiation of the insulin infusion will be recorded, as well as peak glucose level in this time period).

Initiation of beta-blocker: The use of beta-blockers within the 24 hours following surgery if no absolute contraindications were present including: patient with cardiogenic shock (including inotrope or IABP use) or systolic bp $<100 \mathrm{~mm} \mathrm{Hg}$, severe documented COPD, active asthma, bradycardia (HR $<50 / \mathrm{min}$ or symptomatic bradycardia), conduction disorder (ie, second- or third-degree heart block), CHF with left ventricular dysfunction, or physician documentation of other contraindication (ie the preoperative use of beta-blocker will be documented as well as the time and date of initiation of postoperative beta blocker usage).

Continuous telemetry: The use of continuous telemetry monitoring of CABG patients at least until 24 hours before discharge (ie, for patient's with average length of stay).

Aspirin: The prescription of aspirin or antithrombotic (ie, warfarin) in-hospital on the ward and on discharge from hospital or other antiplatelet if the patient has an absolute contraindication (ie, allergy, gastic ulcer, or other physician-stated reason).

Anticoagulation with intravenous heparin at 48 hours after initiation of atrial fibrillation: The initiation of full anticoagulation with heparin, low molecular weight heparin or equivalent (ie, in case of HIT) after sustained atrial fibrillation $>48$ hours.

Beta-blocker: The prescription of a beta-blocker in-hospital on the ward and/or on discharge from hospital except for the following contraindications: systolic bp $<100 \mathrm{~mm} \mathrm{Hg}$, severe docu- mented COPD, active asthma, bradycardia (HR $<50 / \mathrm{min}$ or symptomatic bradycardia), conduction disorder (ie, second- or third-degree heart block), CHF with left ventricular dysfunction, or physician documentation of other contraindication (ie, the preoperative use of beta-blocker will be documented as well as the time and date of initiation of postoperative beta-blocker usage).

Antihyperlipidemic: The prescription of an antihyperlipidemic in-hospital on the ward and/or on discharge from hospital.

ACE inhibitor: The prescription of an ACE inhibitor or angiotensin II receptor blocker in-hospital on the ward and/or on discharge from hospital.

\section{Structure}

*Institutional volume: The institutional yearly volume of CABG procedures.

Nursing to patient ratio in the ICU: The minimum ratio of nurses to patients on average in the ICU for each institution (if this varies greatly - the range and reasons for variation).

Nursing to patient ratio on the ward: The minimum ratio of nurses to patients on average on the ward for each institution (if this varies greatly-the range and reasons for variation).

Daily ward care managed by dedicated nurse practitioners or family physicians: The use of designated care providers on the floor that manage day-to-day postoperative care (ie, the providers involved in care will be recorded; ie. nurse practitioners, family physicians, residents, staff, surgical assistants). 